\title{
Bordetella pertussis infection induces a mucosal IL-17 response and long-lived Th17 and Th1 immune memory cells in nonhuman primates
}

\author{
JM Warfel ${ }^{1}$ and TJ Merkel ${ }^{1}$
}

Despite near universal vaccine coverage, the bacterial pathogen Bordetella pertussis has re-emerged as a major public health concern. We recently developed a baboon (Papio anubis) model of pertussis that provides an excellent model of human pertussis. Using this model, the immune response to pertussis was characterized by measuring cytokines in the nasopharyngeal mucosa of infected baboons. Notably, we observed mucosal expression of interleukin-17 (IL-17) as well as IL-6, IL-23, and several cytokines and chemokines that are orchestrated by IL-17 immune responses. We also found substantial populations of circulating $B$. pertussis-specific Th17 and Th1 cells in convalescent animals $>2$ years postinfection consistent with a role in immunological memory to pertussis. Collectively, these data shed important light on the innate and adaptive immune responses to pertussis in a primate infection model and suggest that Th17 and Th1 immune responses contribute to the immunity conferred by natural pertussis infection.

\section{INTRODUCTION}

Whooping cough is a highly contagious, acute respiratory illness caused by the bacterial pathogen Bordetella pertussis. ${ }^{1,2}$ The introduction of pertussis vaccines in the 1940s and nationwide coverage in excess of $95 \%$ led to a dramatic decrease in the disease. However, for unexplained reasons, pertussis rates in the United States have been steadily rising over the last 30 years in infants, children, and adolescents. ${ }^{3,4}$ With $>27,000$ reported cases in the United States in 2010, the highest number since the 1950s, pertussis is the most common of the vaccinepreventable diseases. ${ }^{5}$ This resurgence is mirrored throughout the industrial world despite similar high rates of vaccination. ${ }^{6-9}$

Several hypotheses have been suggested for the increase in cases but there is no consensus within the scientific community. ${ }^{10}$ One prominent theory is that the vaccine protects against disease but not colonization meaning that the vaccine may not be effective at reducing circulation of pertussis or transmission to naive individuals. Another is that immunity from the acellular vaccine wanes much faster than was previously thought. ${ }^{11}$ Our failure to comprehend and counteract this important public health concern is due in part to gaps in our knowledge of the disease and mechanisms of vaccine-mediated protection. These gaps remain in large part because of the lack of a good animal model of pertussis. Indeed, while a variety of animal models have been used to study the pathogenesis of pertussis including mice, rabbits, guinea-pigs, and newborn piglets, none of these animal models adequately reproduce the full spectrum of the disease observed in humans. ${ }^{12}$ We recently developed a baboon (Papio anubis) model for pertussis that mimics the symptomatology of the human disease. ${ }^{13}$ Pertussis-infected baboons developed clinical disease characterized by nasopharyngeal colonization, leukocytosis, and paroxysmal coughing fits. Using this model, we have also definitively shown that transmission of pertussis occurs via aerosolized respiratory droplets. ${ }^{14}$

One of the major findings from the baboon model is that the host immune response to pertussis infection results in near sterilizing immunity. When convalescent baboons were re-challenged, we observed essentially no colonization of the nasopharynx and no rise in circulating white blood cells. The re-challenged animals displayed a robust boosting of antipertussis toxin serum antibodies but this did not occur until day 5 , suggesting that $\mathrm{T}$-cell memory plays a substantial role in the sterilizing immunity observed in convalescent baboons. ${ }^{13}$ Data

${ }^{1}$ Division of Bacterial, Parasitic and Allergenic Products, Center for Biologics Evaluation and Research, Food and Drug Administration, Bethesda, Maryland, USA. Correspondence: TJ Merkel (tod.merkel@fda.hhs.gov)

Received 26 July 2012; accepted 23 October 2012; published online 28 November 2012. do:10.1038/mi.2012.117 
from the mouse model are consistent with this notion and suggest that both antibodies and T-cell-mediated responses are important for pertussis immunity. ${ }^{15,16}$ This suggests that a thorough understanding of the primate immune response to pertussis may uncover protective mechanisms that could be exploited for designing therapeutics and improved vaccines in humans.

Cytokines play a critical role in the development of CD4 $+\mathrm{T}$ cells that are essential for an effective adaptive immune response. To characterize the immune response to pertussis, we measured cytokines in the nasopharyngeal mucosa of baboons before and during infection. Notably, we observed strong induction of interleukin-6 (IL-6), as well as IL-23, and transient expression of IL-1 $\beta$. This group of cytokines has been implicated in the differentiation of IL-17-producing T cells (Th17 cells) as well as the stimulation of innate $\gamma \delta \mathrm{T}$ cells, both of which are now understood to play critical roles in host immunity to extracellular bacterial and fungal pathogens at mucosal surfaces. ${ }^{17-19}$ Consistent with this role, we detected significant increases in nasopharyngeal IL-17 and several cytokines and chemokines that are orchestrated downstream of IL-17 immune responses. Together, these data suggest that IL-17 plays a prominent role in the local response to pertussis infection in the airways. To explore the adaptive immune response, we isolated peripheral blood mononuclear cells (PBMC) from convalescent animals and stimulated them with heat-killed $B$. pertussis. We detected high concentrations of IL-17 and interferon- $\gamma$ (IFN- $\gamma$ ) in the supernatants. Using ELISPOT we also detected the presence of IL-17- and IFN- $\gamma$-secreting cells in convalescent but not naive animals. These cells are long-lived, having persisted at 2 years postinfection. Using cell separation, we confirmed that these cells are $\mathrm{CD} 4+\mathrm{CD} 95+\mathrm{T}$ cells, consistent with the induction of Th17 and Th1 memory responses to B. pertussis. Collectively, these data shed important light on the innate and adaptive immune responses to pertussis in a primate infection model and suggest that Th17 and Th1 immune responses contribute to the immunity conferred by natural pertussis infection.

\section{RESULTS}

Using the nonhuman primate model recently developed in our laboratory, weanling baboons were infected with the $B$. pertussis clinical isolate $\mathrm{D} 420{ }^{13}$ In order to analyze the primary immune response to pertussis infection, nasopharyngeal washes were collected before and at various time points after challenge and cytokine expression was analyzed using a combination of enzyme-linked immunosorbent assay (ELISA) and a multiplex assay for nonhuman primate cytokines and chemokines. We looked at a panel of cytokines related to Th17 responses (IL-17A, IL-6, IL-1 $\beta$, IL-23), Th17 effector chemokines and cytokines (GCSF (granulocyte colony-stimulating factor), IL-8, MCP-1, MIP-1 $\alpha$ ), Th1 responses (IFN- $\gamma$, IL-12p70), Th2 responses (IL-4, IL-5, IL-13), and other cytokines (MIP-1 $\beta$, GM-CSF, IL-2, IL-15, IL-18, TNF- $\alpha$ ).

\section{B. pertussis infection induces IL-17 response in the nasopharynx}

IL-17 plays a critical role in host immunity to certain extracellular bacterial and fungal pathogens at mucosal surfaces. ${ }^{17,18}$ To determine whether IL-17 was induced in the local mucosa of pertussis-infected baboons, nasopharyngeal washes were collected and analyzed for IL-17 expression by ELISA (Figure 1). Prior to infection, baboons had minimal levels of nasopharyngeal IL-17 and there was little change 2-3 days post-inoculation. However, on days 5 and 7 postinoculation, IL-17 was significantly enhanced with a sevenfold increase over basal levels. By 9-10 days post-inoculation, IL-17 was not significantly different than pre-infection and remained at basal levels through day 16/17.

\section{B. pertussis infection induces IL-6, IL-1 1 , and IL-23 in the nasopharynx}

Because of the IL-17 response observed, we also investigated whether B. pertussis-infected baboons express IL-6, IL-23, and IL-1 $\beta$, cytokines responsible for the initiation and proliferation of Th17 immune responses in humans. ${ }^{20-22}$ Of the entire panel of cytokines tested, the most dramatic induction was seen in IL-6. Samples from pre-infected baboons had low but detectable levels of IL-6. By the first nasopharyngeal wash on day $2 / 3$ postchallenge, the levels had increased $>40$-fold compared with pre-infection levels. On day 5, a 50-fold induction was seen, with IL-6 peaking at nearly $700 \mathrm{pg} / \mathrm{ml}$ in the nasopharyngeal washes. IL-6 levels remained high on day 7 and day $9 / 10$ before dropping on day 12 and remaining low through day $16 / 17$ (Figure 2a). In addition, the IL-12/23p40 subunit was modestly up-regulated through the first week of the infection before peaking at day $9 / 10$ with a threefold increase compared with pre-infected animal samples (Figure $2 \mathbf{b}$ ). Since the p40 subunit can indicate induction of either IL-12p70, which induces the differentiation of Th1 cells, or IL-23, which induces the differentiation of Th17 cells, a subset of post-challenge nasopharyngeal washes were concentrated and analyzed

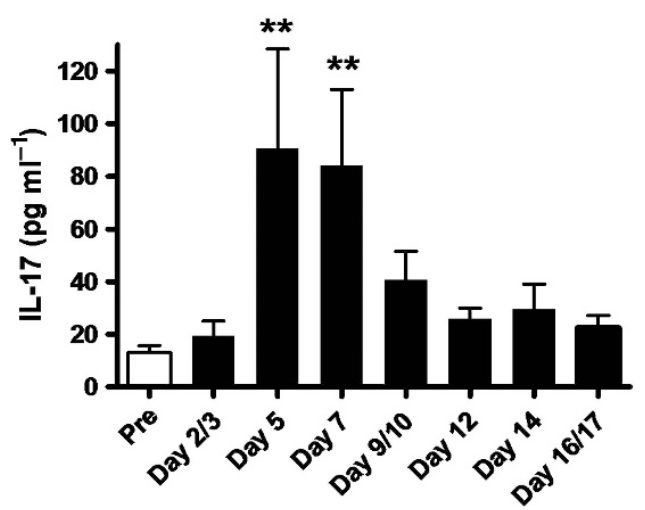

Figure 1 Mucosal interleukin-17 (IL-17) is expressed during B. pertussis infection in baboons. Baboons were inoculated with $B$. pertussis. Nasopharyngeal washes were collected prior to, and on the indicated range of days after inoculation as described in Methods. Samples were analyzed for IL-17A expression by a primate-specific enzyme-linked immunosorbent assay (ELISA) ( $n=6$ for pre-infection and $n=3-5$ for all post-infection time points). ${ }^{\star \star} P<0.01$ vs. pre-infection. 

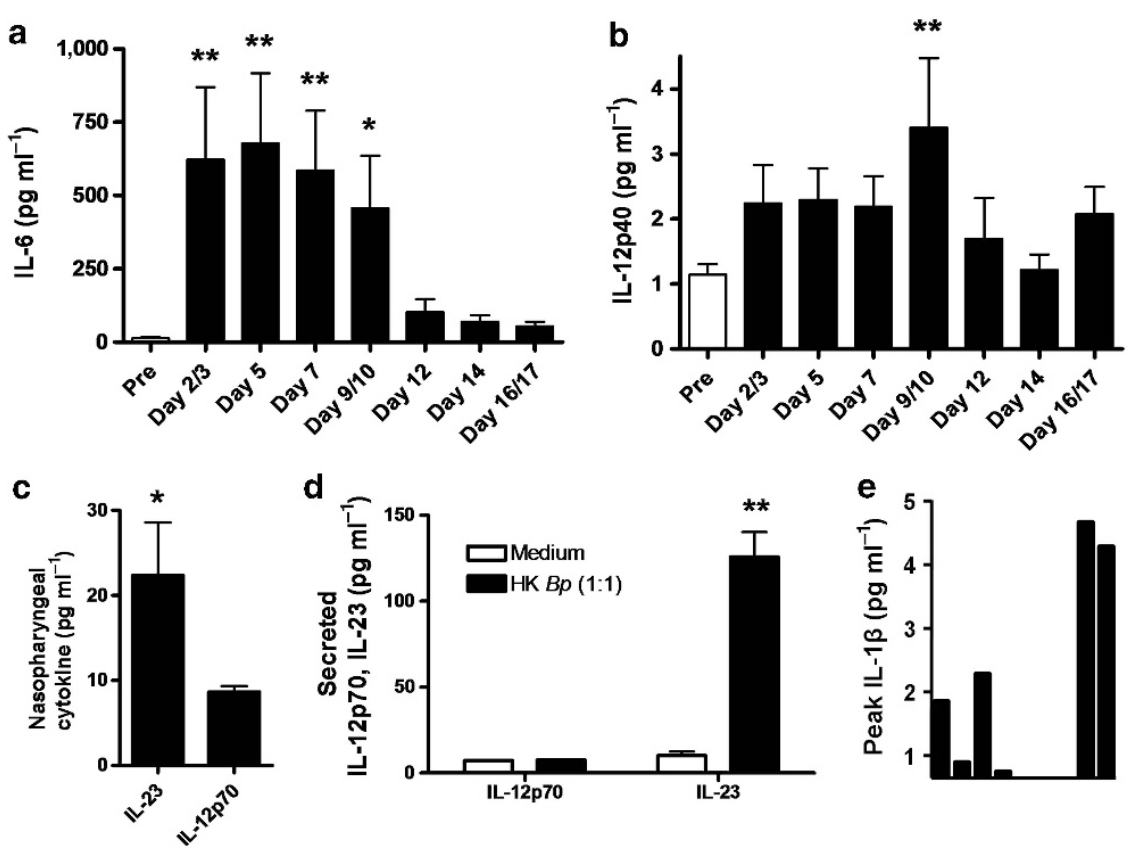

d
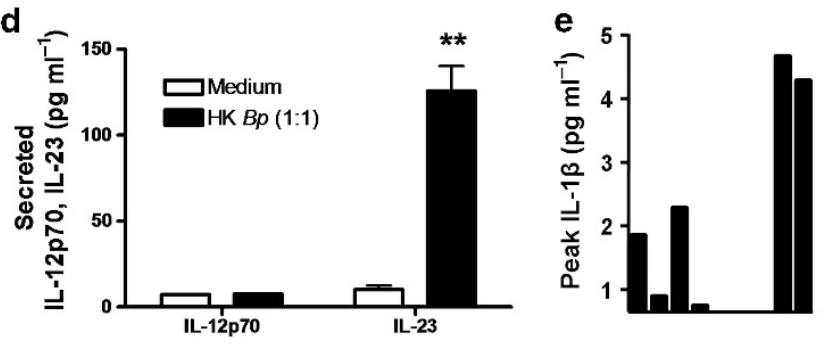

Figure 2 Mucosal expression of interleukin-6 (IL-6), IL-23, and IL-1 $\beta$ in B. pertussis-infected baboons. Nasopharyngeal washes were collected prior to, and on the indicated range of days after inoculation and analyzed by multiplex assay for (a) IL- 6 and (b) IL-12/23p40. Data are presented as mean concentrations with standard error bars ( $n=10$ for pre-infection and $n=7-9$ for all post-infection time points). ${ }^{\star \star} P<0.01,{ }^{*} P<0.05$ vs. pre-infection. (c) A subset of post-challenge nasopharyngeal washes were concentrated and analyzed concurrently by enzyme-linked immunosorbent assay (ELISA) for IL-23 and IL-12p70 as described in the Methods. Data are presented as the mean concentrations with standard error bars $(n=12) .{ }^{*} P<0.05$ for IL-23 vs. IL-12p70 concentrations. (d) Peripheral blood mononuclear cells (PBMC) $\left(10^{6}\right)$ were collected from three baboons, cultured in RPMI medium, and stimulated with heat-killed $B$. pertussis (HK Bp, 1:1 ratio) or medium alone for $72 \mathrm{~h}$. Supernatants were collected and analyzed by ELISA for IL-23 and IL-12p70. Data are presented as the mean concentrations with standard error bars. ${ }^{*} P<0.01$ vs. medium. (e) IL- $1 \beta$ was analyzed by multiplex assay. Shown is the peak post-infection concentration for nine infected baboons. All 10 pre-infection samples analyzed showed no detectable IL-1 $\beta$.

concurrently by ELISA. We detected significantly more IL-23 than IL-12p70, suggesting that the IL-12p40 is indicative of IL-23 (Figure 2c). In further support of these results, we analyzed IL-23 and IL-12p70 secretion from baboon PBMC, which were stimulated ex vivo with heat-killed B. pertussis for $72 \mathrm{~h}$ (1:1 ratio of bacteria to cells). IL-23 was induced 12 -fold in the supernatants from stimulated relative to nonstimulated cells while IL-12p70 was not induced (Figure 2d). IL-1 $\beta$ was not significantly enhanced on any day relative to pre-infection levels. However, we did detect transient IL-1 $\beta$ expression in samples from six out of nine infected baboons on at least 1 day post-infection. In total, 14 out of 55 infected samples were positive for IL-1 $\beta$ (25.5\%) while all 10 pre-infection samples analyzed were below the limit of detection (Figure 2e).

\section{IL-6, IL-23, and IL-1 $\beta$ are part of the primate innate immune response to $B$. pertussis}

To determine whether IL-6, IL-23, and IL-1 $\beta$ represent part of the cytokine profile induced by the primate innate immune response to $B$. pertussis infection, PBMC were isolated from naive baboons and stimulated with 1:1 and 50:1 ratios of heatkilled B. pertussis bacteria to PBMC. As shown in Figure 3, IL-6, IL-1 $\beta$, and IL-23 were induced in a dose-dependent manner. These data suggest that IL- 6 , IL- 23 , and IL- $1 \beta$ are part of the innate immune response to $B$. pertussis in primates.

\section{IL-17-associated chemokines and cytokines are induced following the peak in IL-17}

The protective mechanism of a Th17 response is postulated to be chemokine-mediated recruitment of neutrophils and monocytes to the site of infection. Therefore, the nasopharyngeal wash samples were analyzed for the expression of various Th17 effector molecules: GCSF, IL-8, MCP-1, and MIP$1 \alpha$. GCSF, a cytokine which plays an important role in neutrophil proliferation and differentiation, was enhanced approximately eightfold on day $2 / 3$ and day 5 before peaking at 14-fold on day 7. GCSF levels remained high on day $9 / 10$ and declined thereafter (Figure 4a). Three IL-17 effector chemokines were also upregulated in samples from pertussis-infected animals. IL- 8 and MCP-1 were both trending upward through the first week of the infection and then significantly enhanced on day $9 / 10$ relative to preinfection samples (10-fold enhancement for IL-8 and fivefold for MCP-1) (Figure $\mathbf{4 b}$ and $\mathbf{c}$ ). We also detected significant induction of the chemokine MIP- $1 \alpha$, which was consistently upregulated throughout the course of the infection with significant differences seen on day 5 , day $9 / 10$, and day $16 /$ 17 (four- to fivefold increases) (Figure 4d).

No statistically significant differences were noted in the following cytokines in nasopharyngeal washes of infected animals vs. pre-infection samples: MIP- $1 \beta$, GM-CSF, IFN- $\gamma$, IL-2, IL-4, IL-5, IL-13, IL-15, IL-18, or TNF- $\alpha$ (data not shown). 

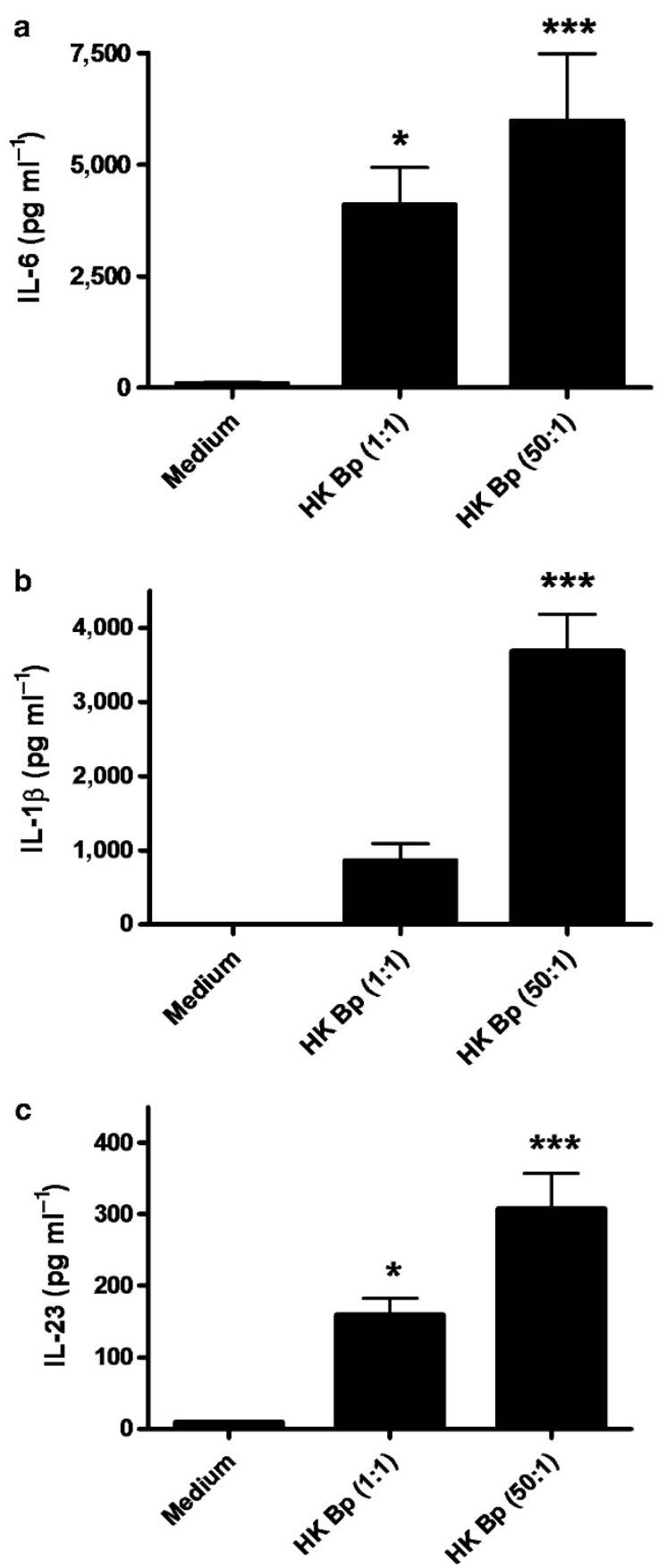

Figure 3 Interleukin-6 (IL-6), IL-23, and IL-1 $\beta$ represent part of the innate cytokine response to $B$. pertussis. Peripheral blood mononuclear cells (PBMC) were collected from naive baboons $(n=3)$ and cultured in RPMI medium. PBMC $\left(10^{6}\right)$ were stimulated with medium alone or with heatkilled $B$. pertussis (HK Bp) at a low concentration (1:1 ratio of bacteria to PBMC) or a high concentration (50:1 ratio). After $72 \mathrm{~h}$, supernatants were collected and analyzed by multiplex assay for IL-6 (a), IL-1 $\beta$ (b) or by IL-23 enzyme-linked immunosorbent assay (ELISA) (c). ${ }^{\star \star \star} P<0.001,{ }^{\star} P<0.05$ vs. medium.

The adaptive immune response to $B$. pertussis is characterized by long-lived Th17 and Th1 memory cells

To determine whether the Th17 cytokine profile induced during a primary infection would carry over to an adaptive immune response, PBMC were isolated from convalescent animals, which had been infected 1-2 months prior and had recently cleared infection. When the convalescent PBMC were incubated with a 1:1 ratio of heat-killed $B$. pertussis, secreted IL-17 was nearly $2,000 \mathrm{pg} / \mathrm{ml}$, and was over $7,000 \mathrm{pg} / \mathrm{ml}$ with the 50:1 ratio (Figure 5a). IL-17 was not induced in similarly stimulated PBMC collected from age-matched naive animals. Since others have shown that the memory response to pertussis in mice and humans includes a Th1 response, we also measured IFN- $\gamma$ expression. ${ }^{15} \mathrm{~W}$ ith the 1:1 ratio of heat-killed B. pertussis, IFN- $\gamma$ was not induced in PBMC from naive or convalescent animals. With the 50:1 ratio, there was minimal innate production of IFN- $\gamma$ from the naive PBMC but $>10$-fold that amount was secreted from convalescent PBMC (Figure 5b). To confirm the presence of cytokine secreting memory immune cells in convalescent animals, we analyzed PBMC by IL-17 and IFN- $\gamma$ ELISPOT. When cells were incubated with a 10:1 ratio of heat-killed $B$. pertussis, IL-17- and IFN- $\gamma$-secreting cells were readily observed in convalescent animals but were absent in naive animals (Figures $5 \mathrm{c}$ and $\mathrm{d}$ ).

To determine the longevity of IL-17 and IFN- $\gamma$-secreting memory cells, PBMC were collected from long-term convalescent animals. Animals were placed into two groups: those that had been infected 6-15 months prior, or 15-24 months prior. For comparison, PBMC were also collected from a group of naive animals with a similar age range. When cells were stimulated and analyzed for secreted cytokines as above, secreted IL-17 was still produced at very high levels in both groups of convalescent PBMC but not in the naive PBMC (Figure 6a). Long-term B. pertussisspecific IFN- $\gamma$ secretion seemed more erratic though it was significantly enhanced in the 15-24 months group (Figure 6b). In a similar fashion, there were significantly more $B$. pertussisspecific IL-17-secreting cells in the 6-15 months and 15-24 months time frames compared with the naive animals while IFN- $\gamma$-secreting cells were observed only in the latter group (Figure 6c).

The fact that immunological memory to $B$. pertussis persists for years in convalescent baboons suggests that the responding cell populations are memory T-helper cells (CD4 + CD95 + ) since most other IL-17-secreting cells are not associated with long-term memory responses. ${ }^{19}$ To test this hypothesis, PBMC from convalescent animals were separated into CD4 - and CD $4+$ populations. When both populations of cells were stimulated with heat-killed $B$. pertussis, IL-17 was secreted only by the CD $4+$ population (Figure 6d). In primates, memory T-helper cells express CD95 on their surface while naive T-helper cells do not. ${ }^{23,24}$ When CD95 + memory T cells were depleted prior to CD4 separation, IL-17 was not secreted by the CD $4+$ CD95 - naive T-cell population (Figure 6d). IFN- $\gamma$ was secreted mainly by stimulated $\mathrm{CD} 4+$ cells but comparatively minor secretion was also seen from stimulated CD4 - cells. However, no IFN- $\gamma$ was secreted by CD $4+$ CD95 - cells (Figure 6e). When PBMC were isolated from naive animals, neither IL-17 nor IFN- $\gamma$ was secreted from any of the separated cell populations (Figure $6 \mathbf{d}$ and e). These data confirm that the $B$. pertussis-responsive 

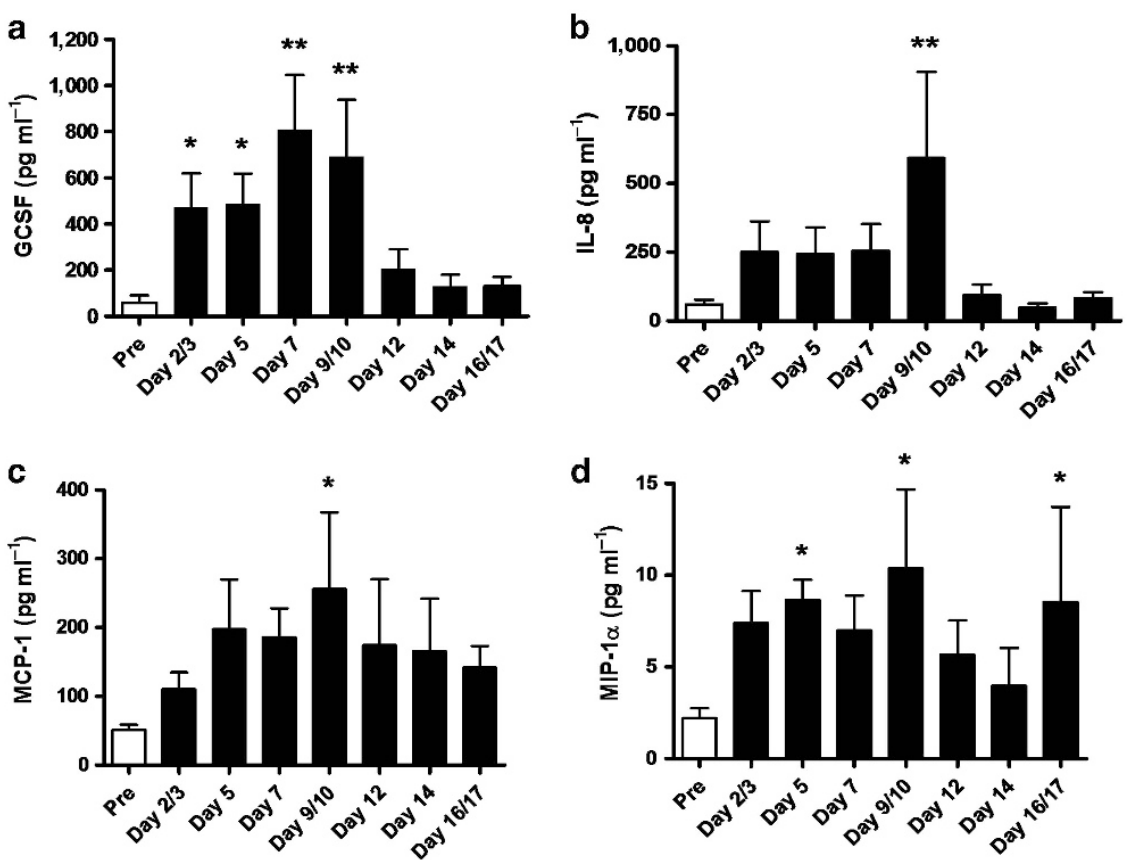

Figure 4 Expression of interleukin-17 (IL-17) effector cytokines and chemokines in B. pertussis-infected baboons. Nasopharyngeal washes were collected prior to, and on the indicated range of days after inoculation and analyzed by multiplex assay for the presence of cytokines and chemokines induced downstream of IL-17: (a) granulocyte colony-stimulating factor (GCSF), (b) IL-8 (CXCL8), (c) MCP-1 (CCL2), and (d) MIP-1 $\alpha$ (CCL3) ( $n=10$ for pre-infection and $n=7$ for all post-infection time points). ${ }^{\star *} P<0.01,{ }^{\star} P<0.05$ vs. pre-infection.

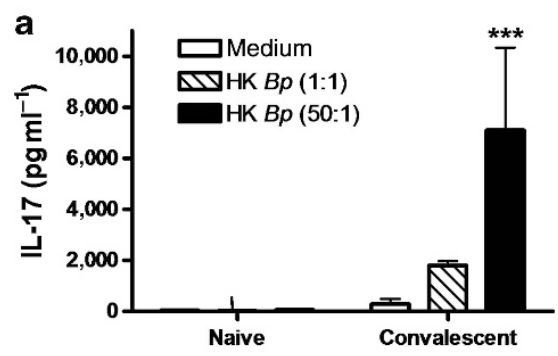

C

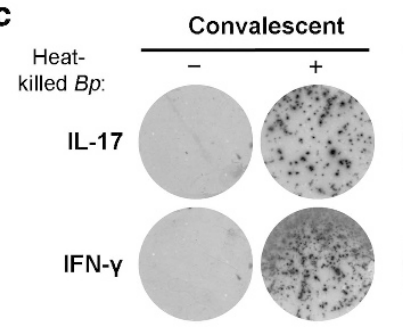

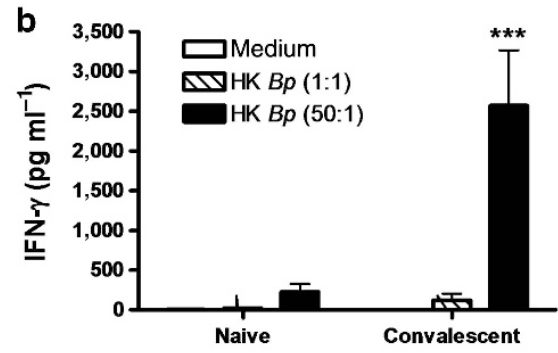

d

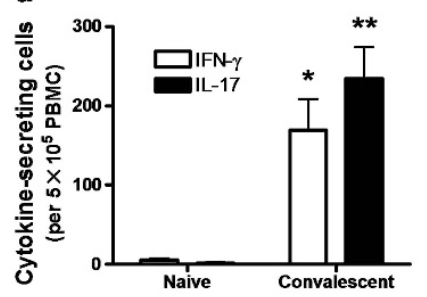

Figure 5 B. pertussis infection induces interleukin-17 (IL-17)- and interferon- $\gamma$ (IFN- $\gamma$ )-secreting adaptive immune cells. Peripheral blood mononuclear cells (PBMC) were collected from naive and recently convalescent baboons (1-2 months post-infection) that had cleared infection and cultured in RMPI medium. PBMC $\left(10^{6}\right)$ were stimulated with medium alone or with heat-killed $B$. pertussis (HK Bp) at a low concentration (1:1 ratio of bacteria to PBMC) or a high concentration (50:1 ratio). After $72 \mathrm{~h}$, supernatants were collected and analyzed by enzyme-linked immunosorbent assay (ELISA) for IL-17 ( $n=4-6)$ (a) and multiplex assay for IFN- $\gamma(n=4-7)\left(\right.$ b). ${ }^{* * *} P<0.001$ vs. convalescent medium treated. (c) PBMC $\left(5 \times 10^{5}\right)$ from naive and convalescent baboons were stimulated with medium alone or with heat-killed $B$. pertussis (10:1 ratio) and IL-17- and IFN- $\gamma$-secreting cells were visualized by ELISPOT. Representative images are shown. (d) B. pertussis-specific IL-17- and IFN- $\gamma$-secreting cells were enumerated from ELISPOT plates as described in Methods $(n=3-4)$. ${ }^{\star \star} P<0.01,{ }^{\star} P<0.05$ vs. naive.

IL-17- and IFN- $\gamma$-secreting immune memory cells in convalescent animals are long-lived CD4 + CD95 + Th17 and Th1 cells.

\section{DISCUSSION}

The lack of an animal model of pertussis that replicates the symptomatology of the human disease has resulted in significant gaps in our knowledge of pertussis pathogenesis and immunity. In order to understand the ongoing rise in pertussis cases and effectively evaluate novel therapeutics and next generation vaccines, a more thorough understanding of the immune response in primates is required. We recently developed a baboon model of pertussis that mimics all the 

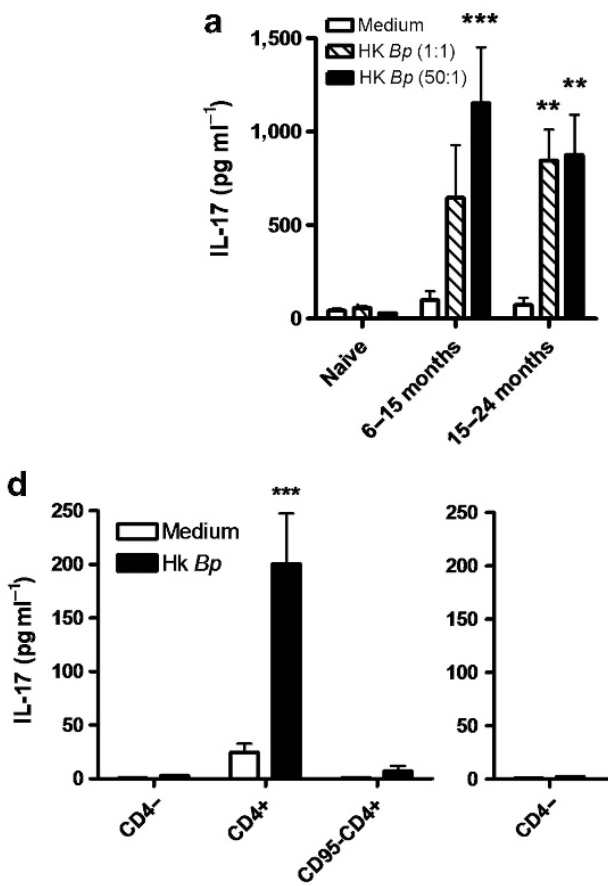

Convalescent animals
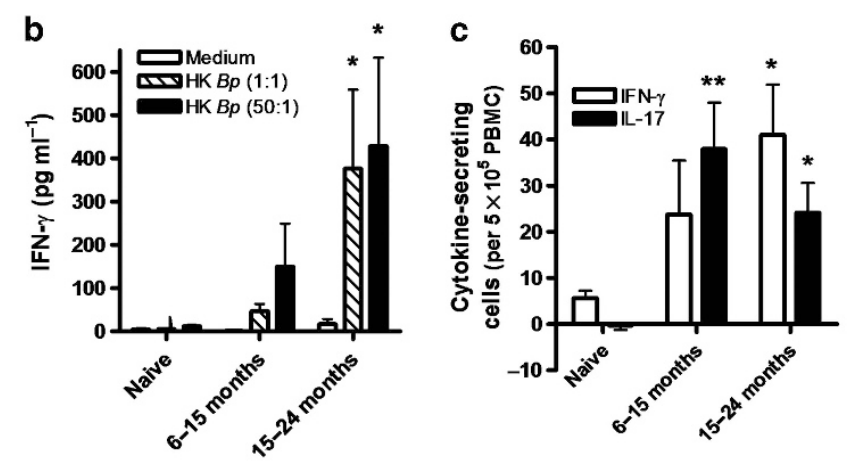

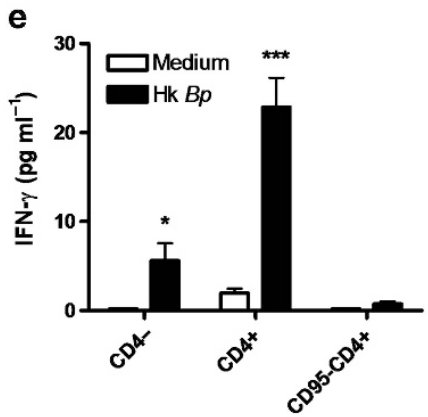

Convalescent animals

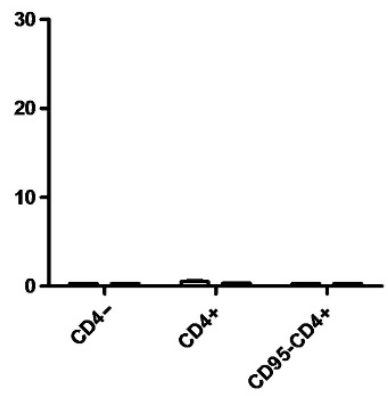

Naive animals

Figure 6 Interleukin-17 (IL-17)- and interferon- $\gamma$ (IFN- $\gamma$ )-secreting immune memory cells after B. pertussis infection are long-lived CD4 + CD95 + Th17 and Th1 cells. Peripheral blood mononuclear cells (PBMC) were collected from naive $(n=3)$ and long-term convalescent baboons (6-15 months, or 15-24 months post-infection, $n=6$ for each group) and cultured in RMPI medium. PBMC $\left(10^{6}\right)$ were incubated with medium or stimulated with heat-killed B. pertussis (HK Bp) at a low concentration (1:1 ratio of bacteria to PBMC) or a high concentration ( $50: 1$ ratio). After $72 \mathrm{~h}$, supernatants were collected and analyzed by enzyme-linked immunosorbent assay (ELISA) for IL-17 (a) and multiplex assay for IFN- $\gamma(\mathbf{b}) .{ }^{* * *} P<0.001$, ${ }^{\star \star} P<0.01$ vs. convalescent medium treated. (c) PBMC $\left(5 \times 10^{5}\right)$ from naive and convalescent baboons were incubated with medium or stimulated with heat-killed $B$. pertussis (10:1 ratio). B. pertussis-specific IL-17- and IFN- $\gamma$-secreting cells were enumerated by ELISPOT as described in Methods. ${ }^{* *} P<0.01,{ }^{*} P<0.05$ vs. naïve. PBMC were collected from naive $(n=3)$ and long-term convalescent baboons $(14-22$ months post-infection, $n=4)$. Nonadherent cells were collected and half were separated using anti-CD4 magnetic particles as described in Methods. The other half of the nonadherent PBMC were depleted of CD95 + cells and then separated with anti-CD4 magnetic particles. CD4 - , CD4 +, and CD95-CD4 + cells were then incubated with medium or stimulated with heat-killed B. pertussis (20:1 ratio of bacteria to pre-fractionated nonadherent PBMC). After $36 \mathrm{~h}$, supernatants were collected and analyzed by ELISA for $\mathrm{IL}-17$ (d) and Milliplex assay for IFN- $\gamma(\mathbf{e})$. For both IL-17 and IFN- $\gamma$, the concentrations $(\mathrm{pg} / \mathrm{ml})$ are presented relative to $10^{6}$ pre-fractionated nonadherent PBMC. ${ }^{* \star \star} P<0.001,{ }^{\star} P<0.05$ vs. convalescent medium treated.

hallmarks of human pertussis including leukocytosis, mucus production, prolonged cough illness, and aerosol transmission from infected to naive hosts. ${ }^{13,14}$

In this study we report that infection of baboons with B. pertussis induces a cytokine profile consistent with the priming of a Th17 response. At the peak of the infection, we observed greater than a 50 -fold increase in nasopharyngeal IL-6. We also observed a significant threefold increase in IL-23 expression and transient induction of IL-1 $\beta$. When PBMC isolated from naive animals were stimulated with heat-killed $B$. pertussis, we detected strong induction of all three of these cytokines, suggesting that this group of cytokines is part of the innate immune response to B. pertussis. In humans and presumably other primates including baboons, IL-6, IL-23, and IL- $1 \beta$ are required for the differentiation of Th17 cells. ${ }^{20,21}$ Consistent with this role, we were able to detect a sevenfold increase in IL-17 expression in nasopharyngeal washes at day 5 and day 7 post-infection compared with pre-infection levels. Future studies are being planned to determine whether the source of this mucosal IL-17 is CD4 + Th17 cells or an innate cell such as $\gamma \delta \mathrm{T}$ cells or NKT cells. ${ }^{19}$
IL-17 immune responses at the respiratory mucosal surfaces are well characterized to play an important role in protecting the host from several extracellular bacterial pathogens. ${ }^{25-27}$ When IL-17 is produced by T cells or innate cells, IL-17 binds to receptors on epithelial cells, endothelial cells and fibroblasts and induces the expression of various cytokines and chemokines that induce neutrophil differentiation and proliferation as well as chemotaxis of neutrophils, macrophages, and lymphocytes to the infected mucosal tissue. ${ }^{17,19,28}$ We detected significant induction of four such compounds induced downstream in the IL-17 pathway: IL-8, GCSF, MCP-1, and MIP$1 \alpha^{25,29,30} \mathrm{IL}-8$ is a potent neutrophil chemokine while GCSF is an essential factor for the proliferation, differentiation, and survival of neutrophils. MCP- 1 and MIP- $1 \alpha$ are chemokines that regulate influx of monocytes and lymphocytes. ${ }^{18,31}$

We also showed that $B$. pertussis-specific IL-17-secreting cells were present in PBMC from convalescent baboons and induced a robust secretion of IL-17 in response to ex vivo stimulation with heat-killed $B$. pertussis. Importantly, the IL-17-secreting immune memory cells were long-lived as we observed $B$. pertussis-specific IL-17 secretion and 
IL-17-secreting cells from animals that had been challenged up to 2 years previously. Using cell separation, we confirmed that these cells are $\mathrm{CD} 4+\mathrm{CD} 95+\mathrm{T}$ cells, consistent with the induction of a Th17 memory response to $B$. pertussis. In addition to providing the first evidence that pertussis infection induces a Th17 response in primates, this is the first demonstration that Th17 cells persist long after pertussis infection and suggest that these cells play an important role in adaptive immunity to pertussis. These findings are consistent with the characterization of human Th17 cells as long-lived effector cells with a high capacity for proliferative selfrenewal. ${ }^{24,32}$

Previous data collected in the mouse model of pertussis as well as from natural human infections in the 1990s and early 2000s suggested that the cell-mediated immune response was geared towards a Th1 profile because of the presence of IFN- $\gamma$ secreting cells. ${ }^{15}$ However, recent data in the mouse model suggest that a major component of the immune response to pertussis infection involves the IL-6 and IL-1-dependent induction of Th17 cells. ${ }^{33,34}$ For example, when IL-17-deficient mice were challenged with $B$. pertussis, the bacterial burden in the lungs at day 7 and day 14 post-infection was 10 - to 100 -fold higher compared with wild-type mice. ${ }^{35}$ In a different study, wild-type mice were treated with a neutralizing antibody to deplete IL-17, resulting in reduced lung chemokine expression and neutrophil recruitment, as well as a significant increase in bacterial burden at day 8 post-infection. ${ }^{36}$ When IL-17R knockout mice were treated with $B$. pertussis, $\sim 100$-fold more $B$. pertussis were recovered from the lungs at day 28 compared with wild-type mice, though there was no difference at earlier time points. ${ }^{34}$

We did not detect induction of the Th2-associated cytokines IL-4, IL-5, or IL-13 either during the primary infection or during the ex vivo recall experiments (data not shown). IFN- $\gamma$ was not detected in the nasopharyngeal washes of infected baboons, but we did identify $B$. pertussis-specific IFN- $\gamma$ secreting memory $\mathrm{CD} 4+\mathrm{CD} 95+\mathrm{T}$ cells in PBMC from convalescent animals. In our cell separation and ex vivo stimulation studies, the majority of the IFN- $\gamma$ was secreted by $\mathrm{CD} 4+$ cells but we also detected some IFN- $\gamma$ secretion from CD4 - PBMC from convalescent but not naive animals, which raises the possibility that there is a relatively minor population of B. pertussis-specific CD8 + memory T cells. Similar to the IL-17-secreting cells, the IFN- $\gamma$-secreting cells appear to be long-lived, having persisted at 2 years post-infection. These data agree well with the paradigm that humans infected with pertussis possess IFN- $\gamma$-secreting T cells but not IL-5-secreting Th2 cells. $^{37-39}$

We did not detect IL-12p70 in vivo or when PBMC were stimulated ex vivo with heat-killed $B$. pertussis, which is contrary to a typical Th1 response where IL-12p70 is an essential factor for differentiation of Th1 cells. ${ }^{40}$ This phenomenon was previously described in an in vitro study by Fedele et al. ${ }^{41,42}$ When treated with $B$. pertussis, human monocyte-derived dendritic cells secreted IL-23 but not IL12p70. However, when the B. pertussis-treated dendritic cells were incubated with naive T cells, both IL-17- and IFN- $\gamma$ secreting $\mathrm{T}$ cells were induced. These puzzling data can possibly be explained by the fact that human Th17 cells can co-express IFN- $\gamma$ raising the possibility that the IFN- $\gamma$-producing memory cells are actually IL-17/IFN- $\gamma$ co-expressing T cells. ${ }^{28} \mathrm{~A}$ recent study found that about $50 \%$ of Staphylococcus aureus-specific human memory Th17 cells co-express IL-17 and IFN- $\gamma$. For the fungal pathogen Candida albicans, nearly all Th17 memory cells were IL-17/IFN- $\gamma$ double positive. ${ }^{20}$

While Th17 cells have been shown to play a critical role in the immune response to primary infection with mucosal pathogens through the recruitment of neutrophils and monocytes, our understanding of their role in adaptive immunity is still evolving. Several recent studies have suggested that Th17 memory cells are attractive targets for vaccines against mucosal pathogens. In a recent study, an extensive panel of antigens from Streptococcus pneumoniae was screened for in vitro induction of IL-17. ${ }^{43}$ When mice were vaccinated with the highest responding antigens, Th17 cells were induced and mice were significantly protected from colonization. This protection was ameliorated when vaccinated mice were treated with neutralizing antibodies for CD4 $+\mathrm{T}$ cells or IL-17 before infection, suggesting a critical role for Th17 cells in immunity induced by this vaccine. When mice were vaccinated with acellular pertussis antigens adjuvanted with Escherichia coli heat-labile enterotoxin instead of alum, the vaccine memory was skewed towards a Th17 response. Importantly, this vaccine was protective in wild-type mice but not IL-17-deficient mice. ${ }^{44}$ Data from the same laboratory have shown that neutralization of IL-17 before challenging wild-type mice with $B$. pertussis reduces the protection afforded by the whole cell vaccine, further suggesting that Th17 memory responses to pertussis antigens are protective. ${ }^{45}$

Because of the acute nature of pertussis infections and because $B$. pertussis has no known animal or environmental reservoir, maintenance of the organism within the human population is thought to require continuous transmission of the disease from infected to naive hosts. This suggests that a successful vaccination program that prevented colonization as well as overt symptoms of pertussis might reduce circulation of B. pertussis in a population. A prominent hypothesis for why the incidence of pertussis is increasing is that the acellular pertussis vaccine does not protect against colonization due to distinct differences in the type of cell-mediated immunity induced by natural infection and vaccination. The present findings, in combination with the studies from the mouse model, suggest that the immune response to natural pertussis infection is skewed towards Th17 and Th1 with no evidence of a Th2 response. ${ }^{33,35,36,45}$ Importantly, this is the first study to show that Th17, in addition to Th1 memory responses to pertussis are induced in primates and are long-lived, suggesting that they play an important role in the sterilizing immunity conferred by natural pertussis infection. Similar to natural infection, the whole cell vaccine in humans has been shown to induce IFN- $\gamma$-secreting memory cells and data from the mouse model additionally suggest that neutralization of IL-17 reduces 
whole cell vaccine-mediated protection. ${ }^{45-49}$ The immunity induced from the acellular vaccine in humans and mice is very different and is characterized by a dominant Th2 response coupled with a weaker Th1 response. ${ }^{46,47,49}$ Using the baboon model, we can assess the ability of vaccination to protect against challenge and determine whether the acellular pertussis vaccines are capable of inducing a similar sterilizing immunity.

\section{METHODS}

Ethics statement. All animal procedures were performed in a facility accredited by the Association for Assessment and Accreditation of Laboratory Animal Care International in accordance with the protocols approved by the CBER Animal Care and Use Committee and the principles outlined in the Guide for the Care and Use of Laboratory Animals by the Institute for Laboratory Animal Resources, National Research Council.

Bacterial strains and media. B. pertussis strain D420 was provided by the Centers for Disease Control and grown on Bordet-Gengou (BG) plates prepared with BG agar (Becton-Dickinson, Sparks, MD, USA) containing $1 \%$ proteose peptone (Becton-Dickinson) and 15\% defibrinated sheep blood. Heat-killed $B$. pertussis was prepared by resuspending bacteria to an OD600 of $0.90\left(5 \times 10^{9} \mathrm{CFU} / \mathrm{ml}\right)$ in PBS and heating at $65^{\circ} \mathrm{C}$ for $30 \mathrm{~min}$.

Infection of baboons. Baboons were obtained from the Oklahoma Baboon Research Resource at the University of Oklahoma Health Sciences Center and housed in a biocontainment unit. ${ }^{14}$ Inoculums were prepared to a concentration between $10^{9}$ and $10^{10}$ bacteria $/ \mathrm{ml}$ as previously described. ${ }^{13}$ Baboons were anesthetized with $10-15 \mathrm{mg} / \mathrm{kg}$ ketamine administered IM. For directly inoculated (index) animals, the pharynx was swabbed with $2 \%$ lidocaine solution and animals were intubated using a $2-3 \mathrm{~mm}$ endotracheal tube to deliver $1 \mathrm{ml}$ of the inoculum to the top of the trachea. A 24 gauge, $3.2 \mathrm{~cm}$ IV catheter (Abbocath) was used to deliver $0.5 \mathrm{ml}$ of inoculum to the back of each naris. Animals were placed in a sitting position for $3 \mathrm{~min}$, returned to their cages, and observed until they recovered from anesthesia.

Collection of nasopharyngeal washes. Baboons were anesthetized with ketamine as described above. The back of each naris was flushed with $1 \mathrm{ml}$ PBS using a 24-guage $/ 3.2 \mathrm{~cm}$ IV catheter. The recovered nasopharyngeal washes from both nares were combined in a tube containing protease inhibitor cocktail (EMD Millipore, Billerica, MA, USA), and stored at $-80^{\circ} \mathrm{C}$ until analyzed for cytokines.

Isolation of PBMC and cell culture. Baboons were anesthetized with ketamine. Peripheral blood was collected into Vacutainer cell preparation tubes with sodium citrate (Becton Dickinson, Franklin Lakes, NJ, USA). Tubes were spun down at $1,800 \mathrm{~g}$ for $20 \mathrm{~min}$ according to the manufacturer's instructions. The plasma layer was removed and the cells were resuspended in complete RPMI-1640 medium supplemented with $10 \%$ sterile heat-inactivated fetal bovine serum, $2 \mathrm{~mm}$ L-glutamine, $25 \mathrm{~mm}$ HEPES, and $1 \times$ antibiotic/antimycotic solution (Thermo Fisher Scientific, Rockford, IL, USA). Cells were washed twice, resuspended in complete RPMI, and incubated at $37^{\circ} \mathrm{C}$ with $5 \%$ $\mathrm{CO}_{2}$. For ex vivo stimulation assays, cells were resuspended at $5 \times 10^{6}$ viable $\mathrm{PBMC} / \mathrm{ml}$ and $200 \mu \mathrm{l}$ were aliquoted into Corning 96-well cell culture round-bottom plates (Corning, NY, USA). Cells were stimulated with medium alone or medium containing heat-killed B. pertussis at a ratio of either 1 bacteria per PBMC or 50 bacteria per PBMC. After $72 \mathrm{~h}$, the cells were spun down at $300 \mathrm{~g}$ for $5 \mathrm{~min}$ and supernatants were recovered, mixed with protease inhibitor cocktail and stored at $-80^{\circ} \mathrm{C}$ until analyzed for cytokines.

Multiplex cytokine assay. Concentrations of IL-1 $\beta$, IL-2, IL-4, IL-5, IL-6, IL-8, IL-12 (p40), IL-13, IL-15, IL-18, MCP-1, MIP-1 $\alpha$, MIP-1 $\beta$, TNF- $\alpha$, GCSF, GM-CSF, and IFN- $\gamma$ were measured using the Milliplex
MAP nonhuman primate kit according to the manufacturer's instructions (Millipore). Briefly, samples were spun down at $10,000 \mathrm{~g}$ for $5 \mathrm{~min}$. Then $25 \mu \mathrm{l}$ of undiluted samples were bound to beads overnight at $4{ }^{\circ} \mathrm{C}$. Each sample was run in duplicate. For every assay, standards for each cytokine were included ranging from 0.64 to $10,000 \mathrm{pg} / \mathrm{ml}$. Following addition of detection antibodies and streptavidin-phycoerythrin, samples were run on the Bio-Plex 200 system (Bio-Rad Laboratories, Hercules, CA, USA). Analysis of cytokine concentrations was performed using Bio-Rad software. For statistical analysis, all samples that were calculated to be below the level of detection were adjusted to the lower limit $(0.64 \mathrm{pg} / \mathrm{ml})$.

ELISA. Nasopharyngeal wash samples and cell culture supernatants were spun down at $10,000 \mathrm{~g}$ for $5 \mathrm{~min}$. IL-17 was quantified using the Ucytech old world monkey IL-17A ELISA antibody pair (Aniara, West Chester, OH, USA) according to the manufacturer's instructions. IL-23 was quantified using a baboon cross-reactive human IL-23 ELISA kit (Mabtech, Mariemont, OH, USA). IL-12p70 was quantified using the Monkey IL-12p70 Platinum ELISA kit (Bender Medsystems, Vienna, Austria). Relative units calculated from the kit were converted to $\mathrm{pg} / \mathrm{ml}$ by comparison to a human IL-12p70 standard curve (Ebioscience, San Diego, CA, USA). For the direct comparison of IL-23 and IL-12p70 expression in vivo, a subset of post-infection nasopharyngeal washes were concentrated with Millipore Amicon Ultra centrifugal filters. The volume of each sample was adjusted to $200 \mu \mathrm{l}$, split in half, and analyzed by ELISA for IL-23 and IL-12p70.

ELISPOT. IL-17- and IFN- $\gamma$-secreting cells were analyzed using Ucytech old world monkey ELISPOT kits according to the manufacturer's instructions. Briefly, $\mathrm{PBMC}$ were isolated as described above and cultured overnight $\left(5 \times 10^{6}\right.$ viable $\left.\mathrm{PBMC} / \mathrm{ml}\right)$ in 24 -well dishes containing RPMI alone or RPMI containing 10 heat-killed B. pertussis bacteria per PBMC to stimulate the cells and deplete monocytes and macrophages. Cells were collected and washed in fresh medium. PBMC were then resuspended in the same type of medium that was used during the pre-stimulation step (i.e., cells that were pre-stimulated with heat-killed $B$. pertussis were resuspended in medium containing heat-killed $B$. pertussis). PBMC $\left(5 \times 10^{5}\right)$ were then aliquoted in triplicate into transparent Nunc Maxisorp 96-well plates pre-coated with anti-monkey IL-17A or anti-monkey IFN- $\gamma$. After $24 \mathrm{~h}, \mathrm{PBMC}$ were washed off and biotinylated detector antibodies were added to the plates and incubated overnight at $4{ }^{\circ} \mathrm{C}$. The wells were then washed and incubated with enzyme-labeled anti-biotin for $1 \mathrm{~h}$ at $37^{\circ} \mathrm{C}$. Subsequently, the wells were washed again and incubated with a substrate solution to develop the silver spots. ELISPOT plates were scanned and analyzed by Cellular Technology Limited (Shaker Heights, OH, USA). To quantify B. pertussis-specific IL-17- and IFN$\gamma$-secreting cells, the number of spots in the nonstimulated wells were subtracted from the number of spots in the wells stimulated with heatkilled B. pertussis.

Cell separation experiments. PBMC were collected from naive and convalescent animals as described above. For each animal, half of the cells were incubated with RPMI alone (nonstimulated control) and the other half were stimulated with RPMI containing 50 heat-killed B. pertussis bacteria per PBMC. After an overnight culture, cells were collected and washed in fresh medium. For each animal and treatment group, the total nonadherent $\mathrm{PBMC}$ were counted and half were depleted of CD95 + cells using biotin mouse anti-human CD95 (DX2 clone, BD Biosciences, San Diego, CA, USA) bound to FlowComp Flexi streptavidin magnetic beads according to the manufacturer's protocol (Invitrogen, Carlsbad, CA, USA). When the beads were separated on a magnet, the CD95 - cells were collected in the supernatants. The remaining total nonadherent cells and the CD95 - cells were then separated using anti-human CD4 magnetic particles (anti-CD4 clone L200, BD Biosciences). Three cell fractions (CD4-, CD4+, $\mathrm{CD} 95-\mathrm{CD} 4+$ ) were resuspended in the same type of medium that 
was used during the pre-separation step (i.e., cells that were stimulated with heat-killed $B$. pertussis were resuspended in RPMI containing heat-killed $B$. pertussis and nonstimulated cells were resuspended in RPMI). The cells were incubated in round-bottom 96-well plates for $36 \mathrm{~h}$. Subsequently, the cells were pelleted and supernatants were collected for IL-17 quantitation by ELISA and IFN- $\gamma$ quantitation by Milliplex assay as described above.

Statistics. Statistical analysis was performed by analysis of variance with post-hoc Student's $t$-test using JMP (version 7) software (SAS Institute, Cary, NC, USA).

\section{ACKNOWLEDGEMENTS}

We thank Dr Joel Beren, Lewis Shankle, Perry Altland, and Ernest Madison for technical assistance. We thank Dr Gary White, Dr Roman Wolf, and Dr James Papin for many helpful discussions and for invaluable help with coordinating the immunological memory experiments. We thank Dr Drusilla Burns, Dr Manuel Osorio, and Dr Brandon Feinen for critical reading of the manuscript. This work was funded by the Food and Drug Administration and NIH/NIAID through Inter-agency agreement \#Y1-AI-1727-01. Baboons were obtained from the Oklahoma Baboon Research Resource. The Oklahoma Baboon Research Resource was supported by Grant Numbers P40RR012317 and 5R24RR016556-10 from the National Institutes of Health National Center For Research Resources.

\section{DISCLOSURE}

The authors declare no conflict of interest.

c 2013 Society for Mucosal Immunology

\section{REFERENCES}

1. Mattoo, S. \& Cherry, J.D. Molecular pathogenesis, epidemiology, and clinical manifestations of respiratory infections due to Bordetella pertussis and other Bordetella subspecies. Clin. Microbiol. Rev. 18 326-382 (2005).

2. Heininger, U. Update on pertussis in children. Expert. Rev. Anti. Infect. Ther. 8, 163-173 (2010).

3. WHO. Pertussis vaccines: WHO position paper. Wkly. Epidemiol. Rec. 85 385-400 (2010).

4. Clark, T.A., Messonnier, N.E. \& Hadler, S.C. Pertussis control: time for something new?. Trends Microbiol. 20, 211-213 (2012).

5. CDC. Provisional cases of selected notifiable diseases. Morbidity Mortality Wkly. Rep. 59, 1706-1716 (2011).

6. Hozbor, D. et al. Pertussis epidemiology in Argentina: trends over 2004-2007. J. Infect. 59, 225-231 (2009).

7. Quinn, H.E. \& McIntyre, P.B. Pertussis epidemiology in Australia over the decade 1995-2005-trends by region and age group. Commun. Dis. Intell. 31, 205-215 (2007).

8. Ntezayabo, B., De Serres, G. \& Duval, B. Pertussis resurgence in Canada largely caused by a cohort effect. Pediatr. Infect. Dis. J. 22, 22-27 (2003).

9. Celentano, L.P. et al. Resurgence of pertussis in Europe. Pediatr. Infect. Dis. J. 24, 761-765 (2005).

10. Friedrich, M.J. Research aims to boost pertussis control. JAMA 306, 27-29 (2011).

11. Witt, M.A., Katz, P.H. \& Witt, D.J. Unexpectedly limited durability of immunity following acellular pertussis vaccination in preadolescents in a North American outbreak. Clin. Infect. Dis. 54, 1730-1735 (2012).

12. Elahi, S., Holmstrom, J. \& Gerdts, V. The benefits of using diverse animal models for studying pertussis. Trends Microbiol. 15, 462-468 (2007).

13. Warfel, J.M., Beren, J., Kelly, V.K., Lee, G. \& Merkel, T.J. Nonhuman primate model of pertussis. Infect. Immun. 80, 1530-1536 (2012).

14. Warfel, J.M., Beren, J. \& Merkel, T.J. Airborne Transmission of Bordetella pertussis. J. Infect. Dis. 206, 902-906 (2012).

15. Higgs, R., Higgins, S.C., Ross, P.J. \& Mills, K.H. Immunity to the respiratory pathogen Bordetella pertussis. Mucosal. Immunol. 5, 485-500 (2012).

16. Leef, M., Elkins, K.L., Barbic, J. \& Shahin, R.D. Protective immunity to Bordetella pertussis requires both $\mathrm{B}$ cells and $\mathrm{CD} 4(+) \mathrm{T}$ cells for key functions other than specific antibody production. J. Exp. Med. 191, 1841-1852 (2000).

17. Kolls, J.K. \& Khader, S.A. The role of Th17 cytokines in primary mucosal immunity. Cytokine Growth Factor Rev. 21, 443-448 (2010).

18. Iwakura, Y., Nakae, S., Saijo, S. \& Ishigame, H. The roles of IL-17A in inflammatory immune responses and host defense against pathogens. Immunol. Rev. 226, 57-79 (2008).

19. Cua, D.J. \& Tato, C.M. Innate IL-17-producing cells: the sentinels of the immune system. Nat. Rev. Immunol. 10, 479-489 (2010).

20. Zielinski, C.E. et al. Pathogen-induced human $\mathrm{TH} 17$ cells produce IFN- $\gamma$ or IL-10 and are regulated by IL-1 $\beta$. Nature 484, 514-518 (2012).

21. McGeachy, M.J. \& Cua, D.J. Th17 cell differentiation: the long and winding road. Immunity 28, 445-453 (2008).

22. Caccamo, N. et al. Differentiation, phenotype, and function of

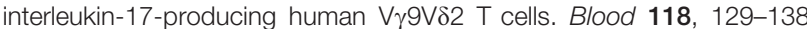
(2011).

23. Pitcher, C.J. et al. Development and homeostasis of $\mathrm{T}$ cell memory in rhesus macaque. J. Immunol. 168, 29-43 (2002).

24. Wei, S., Zhao, E., Kryczek, I. \& Zou, W. Th17 cells have stem cell-like features and promote long-term immunity. Oncoimmunology 1, 516-519 (2012).

25. Ye, P. et al. Interleukin-17 and lung host defense against Klebsiella pneumoniae infection. Am. J. Respir. Cell Mol. Biol. 25, 335-340 (2001).

26. Khader, S.A. et al. $\mathrm{IL}-23$ and $\mathrm{IL}-17$ in the establishment of protective pulmonary CD4 $+\mathrm{T}$ cell responses after vaccination and during Mycobacterium tuberculosis challenge. Nat. Immunol. 8, 369-377 (2007).

27. Cohen, J.M. et al. Protective contributions against invasive Streptococcus pneumoniae pneumonia of antibody and Th17-cell responses to nasopharyngeal colonisation. PLoS One 6, e25558 (2011).

28. Ghoreschi, K., Laurence, A., Yang, X.P., Hirahara, K. \& O'Shea, J.J. T helper 17 cell heterogeneity and pathogenicity in autoimmune disease. Trends Immunol. 32, 395-401 (2011).

29. Happel, K.I. et al. Divergent roles of IL-23 and IL-12 in host defense against Klebsiella pneumoniae. J. Exp. Med. 202, 761-769 (2005).

30. Park, H. et al. A distinct lineage of CD4 T cells regulates tissue inflammation by producing interleukin 17. Nat. Immunol. 6, 1133-1141 (2005).

31. Onishi, R.M. \& Gaffen, S.L. Interleukin-17 and its target genes: mechanisms of interleukin-17 function in disease. Immunology 129, 311-321 (2010).

32. Kryczek, I. et al. Human TH17 cells are long-lived effector memory cells. Sci. Transl. Med. 3, 104ra100 (2011).

33. Zhang, X., Goel, T., Goodfield, L.L., Muse, S.J. \& Harvill, E.T. Decreased leukocyte accumulation and delayed Bordetella pertussis clearance in IL-6 - / - mice. J. Immunol. 186, 4895-4904 (2011).

34. Zhang, X. et al. Interleukin-1 receptor signaling is required to overcome the effects of pertussis toxin and for efficient infection- or vaccination-induced immunity against Bordetella pertussis. Infect. Immun. 79, 527-541 (2011).

35. Dunne, A. et al. Inflammasome activation by adenylate cyclase toxin directs Th17 responses and protection against Bordetella pertussis. J. Immunol. 185, 1711-1719 (2010).

36. Andreasen, C., Powell, D.A. \& Carbonetti, N.H. Pertussis toxin stimulates IL-17 production in response to Bordetella pertussis infection in mice. PLoS One 4, e7079 (2009).

37. Ryan, M. et al. Bordetella pertussis respiratory infection in children is associated with preferential activation of type 1 Thelper cells. J. Infect. Dis. 175, 1246-1250 (1997).

38. Mascart, F. et al. Bordetella pertussis infection in 2-month-old infants promotes type $1 \mathrm{~T}$ cell responses. J Immunol 170, 1504-1509 (2003).

39. Hafler, J.P. \& Pohl-Koppe, A. The cellular immune response to Bordetella pertussis in two children with whooping cough. Eur. J. Med. Res. 3, 523-526 (1998).

40. Watford, W.T., Moriguchi, M., Morinobu, A. \& O'Shea, J.J. The biology of IL-12: coordinating innate and adaptive immune responses. Cytokine Growth Factor Rev. 14, 361-368 (2003).

41. Fedele, G. et al. Bordetella pertussis-infected human monocyte-derived dendritic cells undergo maturation and induce Th1 polarization and interleukin-23 expression. Infect. Immun. 73, 1590-1597 (2005). 


\section{ARTICLES}

42. Fedele, G. et al. Bordetella pertussis commits human dendritic cells to promote a Th1/Th17 response through the activity of adenylate cyclase toxin and MAPK-pathways. PLoS One 5, e8734 (2010).

43. Moffitt, K.L. et al. T(H)17-based vaccine design for prevention of Streptococcus pneumoniae colonization. Cell Host Microbe. 9, 158-165 (2011).

44. Brereton, C.F. et al. Escherichia coli heat-labile enterotoxin promotes protective Th17 responses against infection by driving innate IL-1 and IL-23 production. J. Immunol. 186, 5896-5906 (2011).

45. Higgins, S.C., Jarnicki, A.G., Lavelle, E.C. \& Mills, K.H. TLR4 mediates vaccine-induced protective cellular immunity to Bordetella pertussis: role of IL-17-producing T cells. J. Immunol. 177, 7980-7989 (2006).
46. Ryan, M. et al. Distinct T-cell subtypes induced with whole cell and acellular pertussis vaccines in children. Immunology 93, 1-10 (1998).

47. Dirix, V. et al. Cytokine and antibody profiles in 1-year-old children vaccinated with either acellular or whole-cell pertussis vaccine during infancy. Vaccine 27, 6042-6047 (2009).

48. White, O.J. et al. Th2-polarisation of cellular immune memory to neonatal pertussis vaccination. Vaccine $\mathbf{2 8}, 2648-2652$ (2010).

49. Ausiello, C.M., Urbani, F., la Sala, A., Lande, R. \& Cassone, A. Vaccine- and antigen-dependent type 1 and type 2 cytokine induction after primary vaccination of infants with whole-cell or acellular pertussis vaccines. Infect. Immun. 65, 2168-2174 (1997). 